\title{
Almost Surely Asymptotic Stability of Numerical Solutions for Neutral Stochastic Delay Differential Equations
}

\author{
Zhanhua Yu' ${ }^{1}$ and Mingzhu Liu' ${ }^{2}$ \\ ${ }^{1}$ Department of Mathematics, Harbin Institute of Technology at Weihai, Weihai 264209, China \\ ${ }^{2}$ Department of Mathematics, Harbin Institute of Technology, Harbin 150001, China
}

Correspondence should be addressed to Zhanhua Yu, yuzhanhua@yeah.net

Received 7 March 2011; Accepted 11 April 2011

Academic Editor: Her-Terng Yau

Copyright (C) 2011 Z. Yu and M. Liu. This is an open access article distributed under the Creative Commons Attribution License, which permits unrestricted use, distribution, and reproduction in any medium, provided the original work is properly cited.

We investigate the almost surely asymptotic stability of Euler-type methods for neutral stochastic delay differential equations (NSDDEs) using the discrete semimartingale convergence theorem. It is shown that the Euler method and the backward Euler method can reproduce the almost surely asymptotic stability of exact solutions to NSDDEs under additional conditions. Numerical examples are demonstrated to illustrate the effectiveness of our theoretical results.

\section{Introduction}

The neutral stochastic delay differential equation (NSDDE) has attracted much more attention, and much work (see [1-4]) has been done. For example, Mao [2] studied the existence and uniqueness, moment and pathwise estimates, and the exponential stability of the solution to the NSDDE. Moreover, Mao et al. [4] studied the almost surely asymptotic stability of the NEDDE with Markovian switching:

$$
d[x(t)-N(x(t-\tau), r(t))]=f(t, x(t), x(t-\tau), r(t)) d t+g(t, x(t), x(t-\tau), r(t)) d B(t) .
$$

Since most NSDDEs cannot be solved explicitly, numerical solutions have become an important issue in the study of NSDDEs. Convergence analysis of numerical methods for NSDDEs can be found in [5-7]. On the other hand, stability theory of numerical solutions is one of the fundamental research topics in the numerical analysis. For stochastic differential equations (SDEs) as well as stochastic delay differential equations (SDDEs), 
moment stability and asymptotic stability of numerical solutions have received much more attention (e.g., [8-13] for moment stability and [12-14] for asymptotic stability). Recently, Wang and Chen [15] studied the mean-square stability of the semi-implicit Euler method for NSDDEs. We aim in this paper to study the almost surely asymptotic stability of Euler-type methods for NSDDEs using the discrete semimartingale convergence theorem. The discrete semimartingale convergence theorem (cf. $[16,17]$ ) plays an important role in the almost surely asymptotic stability analysis of numerical solutions to SDEs and SDDEs [17-19]. Using the discrete semimartingale convergence theorem, we show that Euler-type methods for NSDDEs can preserve the almost surely asymptotic stability of exact solutions under additional conditions.

In Section 2, we introduce some necessary notations and state the discrete semimartingale convergence theorem as a lemma. In Section 3, we study the almost surely asymptotic stability of exact solutions to NSDDEs. Section 4 gives the almost surely asymptotic stability of the Euler method. In Section 5, we discuss the almost surely asymptotic stability of the backward Euler method. Numerical experiments are presented in Section 6.

\section{Preliminaries}

Throughout this paper, unless otherwise specified, we use the following notations. Let $\left(\Omega, \mathcal{F},\left\{\mathcal{F}_{t}\right\}_{t \geq 0}, P\right)$ be a complete probability space with filtration $\left\{\mathcal{F}_{t}\right\}_{t \geq 0}$ satisfying the usual conditions (i.e., it is right continuous and $\mathscr{F}_{0}$ contains all $P$-null sets). $B(t)$ is a scalar Brownian motion defined on the probability space. $|\cdot|$ denotes the Euclidean norm in $R^{n}$. The inner product of $x, y$ in $R^{n}$ is denoted by $\langle x, y\rangle$ or $x^{T} y$. If $A$ is a vector or matrix, its transpose is denoted by $A^{T}$. If $A$ is a matrix, its trace norm is denoted by $|A|=\sqrt{\operatorname{trace}\left(A^{T} A\right)}$. Let $\tau>0$ and $C\left([-\tau, 0] ; R^{n}\right)$ denote the family of all continuous $R^{n}$-valued functions on $[-\tau, 0]$. Let $C_{\mp_{0}}^{b}\left([-\tau, 0] ; R^{n}\right)$ be the family of all $\Psi_{0}$-measurable bounded $C\left([-\tau, 0] ; R^{n}\right)$-valued random variables $\xi=\{\xi(\theta):-\tau \leq \theta \leq 0\}$.

Consider an $n$-dimensional NSDDE

$$
d[x(t)-N(x(t-\tau))]=f(t, x(t), x(t-\tau)) d t+g(t, x(t), x(t-\tau)) d B(t),
$$

on $t \geq 0$ with initial data $\{x(\theta):-\tau \leq \theta \leq 0\}=\xi \in C_{\mathcal{\mp}_{0}}^{b}\left([-\tau, 0] ; R^{n}\right)$. Here $N: R^{n} \rightarrow R^{n}$, $f: R^{+} \times R^{n} \times R^{n} \rightarrow R^{n}$, and $g: R^{+} \times R^{n} \times R^{n} \rightarrow R^{n}$.

Let $C^{1,2}\left(R^{+} \times R^{n} ; R^{+}\right)$denote the family of all nonnegative functions $V(t, x)$ on $R^{+} \times R^{n}$ which are continuously once differentiable in $t$ and twice differentiable in $x$. For each $V \in$ $C^{1,2}\left(R^{+} \times R^{n} ; R^{+}\right)$, define an operator LV from $R^{+} \times R^{n} \times R^{n}$ to $R$ by

$$
\begin{aligned}
\mathrm{LV}(t, x, y)= & V_{t}(t, x-N(y))+V_{x}(t, x-N(y)) f(t, x, y) \\
& +\frac{1}{2} \operatorname{trace}\left[g^{T}(t, x, y) V_{x x}(t, x-N(y)) g(t, x, y)\right],
\end{aligned}
$$

where

$$
V_{t}(t, x)=\frac{\partial V(t, x)}{\partial t}, \quad V_{x}(t, x)=\left(\frac{\partial V(t, x)}{\partial x_{1}}, \ldots, \frac{\partial V(t, x)}{\partial x_{n}}\right), \quad V_{x x}(t, x)=\left(\frac{\partial^{2} V(t, x)}{\partial x_{i} \partial x_{j}}\right)_{n \times n}
$$


As a standing hypothesis, we impose the following assumption on the coefficients $N, f$, and $g$.

Assumption 2.1. Assume that both $f$ and $g$ satisfy the local Lipschitz condition. That is, for each integer $i>0$, there exists a positive constant $K_{i}$ such that

$$
|f(t, x, y)-f(t, \bar{x}, \bar{y})|^{2} \vee|g(t, x, y)-g(t, \bar{x}, \bar{y})|^{2} \leq K_{i}\left(|x-\bar{x}|^{2}+|y-\bar{y}|^{2}\right)
$$

for $x, y, \bar{x}, \bar{y} \in R^{n}$ with $|x| \vee|\bar{x}| \vee|y| \vee|\bar{y}| \leq i$ and $t \in R^{+}$. Assume also that there is a constant $\kappa \in(0,1)$ such that

$$
|N(x)-N(y)| \leq \kappa|x-y|, \quad \forall x, y \in R^{n}
$$

Assume moreover that for all $t \in R^{+}$,

$$
N(0)=0, \quad f(t, 0,0)=0, \quad g(t, 0,0)=0 .
$$

The following discrete semimartingale convergence theorem (cf. $[16,17])$ will play an important role in this paper.

Lemma 2.2. Let $\left\{A_{i}\right\}$ and $\left\{U_{i}\right\}$ be two sequences of nonnegative random variables such that both $A_{i}$ and $U_{i}$ are $\mathcal{F}_{i}$-measurable for $i=1,2, \ldots$, and $A_{0}=U_{0}=0$ a.s. Let $M_{i}$ be a real-valued local martingale with $M_{0}=0$ a.s. Let $\zeta$ be a nonnegative $\mathcal{F}_{0}$-measurable random variable. Assume that $\left\{X_{i}\right\}$ is a nonnegative semimartingale with the Doob-Mayer decomposition

$$
X_{i}=\zeta+A_{i}-U_{i}+M_{i}
$$

If $\lim _{i \rightarrow \infty} A_{i}<\infty$ a.s., then for almost all $\omega \in \Omega$

$$
\lim _{i \rightarrow \infty} X_{i}<\infty, \quad \lim _{i \rightarrow \infty} U_{i}<\infty,
$$

that is, both $X_{i}$ and $U_{i}$ converge to finite random variables.

\section{Almost Surely Asymptotic Stability of the Exact Solution}

In this section, we will study the almost surely asymptotic stability of exact solutions to (2.1). To be precise, let us give the definition on the almost surely asymptotic stability of exact solutions.

Definition 3.1. The solution $x(t)$ to (2.1) is said to be almost surely asymptotically stable if

$$
\lim _{t \rightarrow \infty} x(t)=0 \quad \text { a.s. }
$$

for any initial data $\xi \in C_{\mathcal{\mp}_{0}}^{b}\left([-\tau, 0] ; R^{n}\right)$. 
Theorem 3.2. Let Assumption 2.1 hold. Assume that there are four positive constants $\lambda_{1}-\lambda_{4}$ such that

$$
\begin{gathered}
2(x-N(y))^{T} f(t, x, y) \leq-\lambda_{1}|x|^{2}+\lambda_{2}|y|^{2}, \\
|g(t, x, y)|^{2} \leq \lambda_{3}|x|^{2}+\lambda_{4}|y|^{2}
\end{gathered}
$$

for $t \geq 0$ and $x, y \in R^{n}$. If

$$
\lambda_{1}-\lambda_{3}>\lambda_{2}+\lambda_{4}
$$

then, for any initial data $\xi \in C_{q_{0}}^{b}\left([-\tau, 0] ; R^{n}\right)$, there exists a unique global solution $x(t)$ to $(2.1)$ and the solution $x(t)$ is almost surely asymptotically stable.

Proof. Let $U(t, x)=|x|^{2}$. Using (3.2) and (3.3), we have

$$
\begin{aligned}
\mathrm{LU}(t, x, y) & =2(x-N(y))^{T} f(t, x, y)+|g(t, x, y)|^{2} \\
& \leq-\left(\lambda_{1}-\lambda_{3}\right)|x|^{2}+\left(\lambda_{2}+\lambda_{4}\right)|y|^{2} \\
& \leq-\left(\lambda_{1}-\lambda_{3}\right)|x|^{2}+\left(\lambda_{1}-\lambda_{3}\right)|y|^{2} .
\end{aligned}
$$

Then, from Theorem 3.1 in [4], we conclude that there exists a unique global solution $x(t)$ to (2.1) for any initial data $\xi \in C_{q_{0}}^{b}\left([-\tau, 0] ; R^{n}\right)$. According to (3.3), there is a constant $\bar{\alpha}>0$ such that

$$
\lambda_{1}-\lambda_{3}-2 \alpha \geq\left(\lambda_{2}+\lambda_{4}+2 \alpha\right) e^{\alpha \tau} \quad \forall 0 \leq \alpha \leq \bar{\alpha} .
$$

Let $V(t, x)=e^{\alpha t} U(t, x)$. Here $\alpha \in(0, \bar{\alpha}] \cap(0,(2 / \tau) \log (1 / \kappa))$. Then

$$
\begin{aligned}
\operatorname{LV}(t, x, y) & =e^{\alpha t}(\alpha U(t, x-N(y)))+\operatorname{LU}(t, x) \\
& \leq e^{\alpha t}\left[\alpha|x-N(y)|^{2}-\left(\lambda_{1}-\lambda_{3}\right)|x|^{2}+\left(\lambda_{2}+\lambda_{4}\right)|y|^{2}\right] \\
& \leq e^{\alpha t}\left[-\left(\lambda_{1}-\lambda_{3}-2 \alpha\right)|x|^{2}+\left(\lambda_{2}+\lambda_{4}+2 \alpha\right)|y|^{2}\right] \\
& \leq-W(t, x)+W(t-\tau, y),
\end{aligned}
$$

where $W(t, x)=\left(\lambda_{1}-\lambda_{3}-2 \alpha\right) e^{\alpha t}|x|^{2}$. By Theorem 4.1 in [4], we can obtain that the solution $x(t)$ is almost surely asymptotically stable. The proof is completed.

Theorem 3.2 gives sufficient conditions of the almost surely asymptotic stability of the NSDDE (2.1). Based on these sufficient conditions, we will investigate the almost surely asymptotic stability of Euler-type methods in the following sections. 


\section{Stability of the Euler Method}

Applying the Euler method (EM) to (2.1) yields

$$
\begin{gathered}
X_{k+1}-N\left(X_{k+1-m}\right)=X_{k}-N\left(X_{k-m}\right)+f\left(k h, X_{k}, X_{k-m}\right) h \\
+g\left(k h, X_{k}, X_{k-m}\right) \Delta B_{k}, \quad k=0,1,2, \ldots, \\
X_{k}=\xi(k h), \quad k=-m,-m+1, \ldots, 0 .
\end{gathered}
$$

Here $h=\tau / m$ ( $m$ is an positive integer) is the stepsize, and $\Delta B_{k}=B((k+1) h)-B(k h)$ represents the Browian motion increment. To be precise, let us introduce the definition on the almost surely asymptotic stability of numerical solutions.

Definition 4.1. The numerical solution $X_{k}$ to (2.1) is said to be almost surely asymptotically stable if

$$
\lim _{k \rightarrow \infty} X_{k}=0 \text { a.s. }
$$

for any bounded variables $\xi(k h), k=-m,-m+1, \ldots, 0$.

Theorem 4.2. Let conditions (3.2)-(3.3) hold. Assume that $f$ satisfies the linear growth condition, namely, there exists a positive constant $L$ such that

$$
|f(t, x, y)|^{2} \leq L\left(|x|^{2}+|y|^{2}\right)
$$

Then there exists a $h_{0}>0$ such that if $h<h_{0}$, then for any given finite-valued $\mathcal{F}_{0}$-measurable random variables $\xi(k h), k=-m,-m+1, \ldots, 0$, the EM approximate solution (4.1) is almost surely asymptotically stable.

Proof. Let $Y_{k}=X_{k}-N\left(X_{k-m}\right)$. Then, it follows from (4.1) that

$$
Y_{k+1}=Y_{k}+f\left(k h, X_{k}, X_{k-m}\right) h+g\left(k h, X_{k}, X_{k-m}\right) \Delta B_{k}, \quad k=0,1,2, \ldots
$$

Squaring both sides of (4.4), we have

$$
\begin{aligned}
\left|Y_{k+1}\right|^{2}= & \left|Y_{k}\right|^{2}+\left|f\left(k h, X_{k}, X_{k-m}\right)\right|^{2} h^{2}+\left|g\left(k h, X_{k}, X_{k-m}\right)\right|^{2} h \\
& +2\left\langle Y_{k}, f\left(k h, X_{k}, X_{k-m}\right)\right\rangle h+2\left\langle Y_{k}, g\left(k h, X_{k}, X_{k-m}\right)\right\rangle \Delta B_{k} \\
& +2 h\left\langle f\left(k h, X_{k}, X_{k-m}\right), g\left(k h, X_{k}, X_{k-m}\right)\right\rangle \Delta B_{k} \\
& +\left|g\left(k h, X_{k}, X_{k-m}\right)\right|^{2}\left(\Delta B_{k}^{2}-h\right) .
\end{aligned}
$$


Using (3.2) and (4.3), we can obtain that

$$
\begin{aligned}
\left|Y_{k+1}\right|^{2} \leq & \left|Y_{k}\right|^{2}+L\left(\left|X_{k}\right|^{2}+\left|X_{k-m}\right|^{2}\right) h^{2}+\left(\lambda_{3}\left|X_{k}\right|^{2}+\lambda_{4}\left|X_{k-m}\right|^{2}\right) h \\
& +\left(-\lambda_{1}\left|X_{k}\right|^{2}+\lambda_{2}\left|X_{k-m}\right|^{2}\right) h+m_{k}
\end{aligned}
$$

where

$$
\begin{aligned}
m_{k}= & 2\left\langle Y_{k}, g\left(k h, X_{k}, X_{k-m}\right)\right\rangle \Delta B_{k}+2 h\left\langle f\left(k h, X_{k}, X_{k-m}\right), g\left(k h, X_{k}, X_{k-m}\right)\right\rangle \Delta B_{k} \\
& +\left|g\left(k h, X_{k}, X_{k-m}\right)\right|^{2}\left(\Delta B_{k}^{2}-h\right) .
\end{aligned}
$$

It therefore follows that

$$
\left|Y_{k+1}\right|^{2}-\left|Y_{k}\right|^{2} \leq-\left(\lambda_{1}-\lambda_{3}-L h\right) h\left|X_{k}\right|^{2}+\left(\lambda_{2}+\lambda_{4}+L h\right) h\left|X_{k-m}\right|^{2}+m_{k}
$$

which implies that

$$
\left|Y_{k}\right|^{2}-\left|Y_{0}\right|^{2} \leq-\left(\lambda_{1}-\lambda_{3}-L h\right) h \sum_{i=0}^{k-1}\left|X_{i}\right|^{2}+\left(\lambda_{2}+\lambda_{4}+L h\right) h \sum_{i=0}^{k-1}\left|X_{i-m}\right|^{2}+\sum_{i=0}^{k-1} m_{i} .
$$

Note that

$$
\sum_{i=0}^{k-1}\left|X_{i-m}\right|^{2}=\sum_{i=-m}^{-1}\left|X_{i}\right|^{2}+\sum_{i=0}^{k-m-1}\left|X_{i}\right|^{2}
$$

Then, we have

$$
\begin{aligned}
\left|Y_{k}\right|^{2}+\left(\lambda_{2}+\lambda_{4}+L h\right) h \sum_{i=k-m}^{k-1}\left|X_{i}\right|^{2} \leq & \left|Y_{0}\right|^{2}+\left(\lambda_{2}+\lambda_{4}+L h\right) h \sum_{i=-m}^{-1}\left|X_{i}\right|^{2} \\
& -\left(\lambda_{1}-\lambda_{3}-\lambda_{2}-\lambda_{4}-2 L h\right) h \sum_{i=0}^{k-1}\left|X_{i}\right|^{2}+M_{k},
\end{aligned}
$$

where $M_{k}=\sum_{i=0}^{k-1} m_{i}$. By [18], $M_{k}$ is a martingale with $M_{0}=0$. From (3.3), we obtain that

$$
\lambda_{1}-\lambda_{3}-\lambda_{2}-\lambda_{4}-2 L h>0, \quad \text { as } 0<h<h_{0},
$$

where $h_{0}=\left(\lambda_{1}-\lambda_{3}-\lambda_{2}-\lambda_{4}\right) / 2 L$. Hence, from Lemma 2.2, we therefore have

$$
\lim _{k \rightarrow \infty} \sum_{i=0}^{k-1}\left|X_{i}\right|^{2}<\infty \quad \text { a.s., } 0<h<h_{0}
$$


Then, we conclude that

$$
\lim _{k \rightarrow \infty}\left|X_{k}\right|^{2}=0 \quad \text { a.s., } 0<h<h_{0}
$$

The proof is completed.

Theorem 4.2 shows that if the coefficient $f$ obeys the linear growth condition, in addition to the conditions imposed in Theorem 3.2, then the EM approximate solution (4.1) reproduces the almost surely asymptotic stability of exact solutions to (2.1) for sufficiently small stepsize.

\section{Stability of the Backward Euler Method}

Applying the backward Euler method (BEM) to (2.1) yields

$$
\begin{gathered}
X_{k+1}-N\left(X_{k+1-m}\right)=X_{k}-N\left(X_{k-m}\right)+f\left((k+1) h, X_{k+1}, X_{k+1-m}\right) h \\
+g\left(k h, X_{k}, X_{k-m}\right) \Delta B_{k}, \quad k=0,1,2, \ldots, \\
X_{k}=\xi(k h), \quad k=-m,-m+1, \ldots, 0 .
\end{gathered}
$$

As a standing hypothesis, we assume that the BEM (5.1) is well defined. The following theorem shows that if the above assumption and the conditions imposed in Theorem 3.2 hold, then the BEM approximate solution (5.1) inherits the almost surely asymptotic stability of exact solutions to (2.1) without any stepsize restriction.

Theorem 5.1. Let conditions (3.2)-(3.3) hold. Then for any given finite-valued $\mathcal{F}_{0}$-measurable random variables $\xi(k h), k=-m,-m+1, \ldots, 0$, the BEM approximate solution (5.1) is almost surely asymptotically stable.

Proof. Let $Y_{k}=X_{k}-N\left(X_{k-m}\right)$. Then, it follows from (5.1) that

$$
Y_{k+1}-f\left((k+1) h, X_{k+1}, X_{k+1-m}\right) h=Y_{k}+g\left(k h, X_{k}, X_{k-m}\right) \Delta B_{k}, \quad k=0,1,2, \ldots
$$

Squaring both sides of (5.2), we have

$$
\begin{aligned}
\left|Y_{k+1}\right|^{2} & +\left|f\left((k+1) h, X_{k+1}, X_{k+1-m}\right)\right|^{2} h^{2} \\
= & \left|Y_{k}\right|^{2}+2\left\langle Y_{k+1}, f\left((k+1) h, X_{k+1}, X_{k+1-m}\right)\right\rangle h+\left|g\left(k h, X_{k}, X_{k-m}\right)\right|^{2} h \\
& +2\left\langle Y_{k}, g\left(k h, X_{k}, X_{k-m}\right)\right\rangle \Delta B_{k}+\left|g\left(k h, X_{k}, X_{k-m}\right)\right|^{2}\left(\Delta B_{k}^{2}-h\right) .
\end{aligned}
$$

Using (3.2), we can obtain that

$$
\left|Y_{k+1}\right|^{2} \leq\left|Y_{k}\right|^{2}+\left(-\lambda_{1}\left|X_{k+1}\right|^{2}+\lambda_{2}\left|X_{k+1-m}\right|^{2}\right) h+\left(\lambda_{3}\left|X_{k}\right|^{2}+\lambda_{4}\left|X_{k-m}\right|^{2}\right) h+m_{k}
$$


where

$$
m_{k}=2\left\langle Y_{k}, g\left(k h, X_{k}, X_{k-m}\right)\right\rangle \Delta B_{k}+\left|g\left(k h, X_{k}, X_{k-m}\right)\right|^{2}\left(\Delta B_{k}^{2}-h\right) .
$$

It therefore follows that

$$
\left|Y_{k+1}\right|^{2}-\left|Y_{k}\right|^{2} \leq-\lambda_{1} h\left|X_{k+1}\right|^{2}+\lambda_{3} h\left|X_{k}\right|^{2}+\lambda_{2} h\left|X_{k+1-m}\right|^{2}+\lambda_{4} h\left|X_{k-m}\right|^{2}+m_{k}
$$

which implies that

$$
\begin{aligned}
\left|Y_{k}\right|^{2}-\left|Y_{0}\right|^{2} \leq & -\lambda_{1} h \sum_{i=0}^{k-1}\left|X_{i+1}\right|^{2}+\lambda_{3} h \sum_{i=0}^{k-1}\left|X_{i}\right|^{2}+\lambda_{2} h \sum_{i=0}^{k-1}\left|X_{i+1-m}\right|^{2} \\
& +\lambda_{4} h \sum_{i=0}^{k-1}\left|X_{i-m}\right|^{2}+\sum_{i=0}^{k-1} m_{i} .
\end{aligned}
$$

Note that

$$
\begin{aligned}
& \sum_{i=0}^{k-1}\left|X_{i-m}\right|^{2}=\sum_{i=-m}^{-1}\left|X_{i}\right|^{2}+\sum_{i=0}^{k-m-1}\left|X_{i}\right|^{2} \\
& \sum_{i=0}^{k-1}\left|X_{i+1-m}\right|^{2}=\sum_{i=-m+1}^{-1}\left|X_{i}\right|^{2}+\sum_{i=0}^{k-m}\left|X_{i}\right|^{2} .
\end{aligned}
$$

Then, we have

$$
\begin{aligned}
\left|Y_{k}\right|^{2} \leq & \left|Y_{0}\right|^{2}+\lambda_{2} h \sum_{i=-m+1}^{-1}\left|X_{i}\right|^{2}+\lambda_{4} h \sum_{i=-m}^{-1}\left|X_{i}\right|^{2} \\
& -\lambda_{1} h \sum_{i=1}^{k}\left|X_{i}\right|^{2}+\lambda_{3} h \sum_{i=0}^{k-1}\left|X_{i}\right|^{2} \\
& +\lambda_{2} h \sum_{i=0}^{k-m}\left|X_{i}\right|^{2}+\lambda_{4} h \sum_{i=0}^{k-m-1}\left|X_{i}\right|^{2}+\sum_{i=0}^{k-1} m_{i} .
\end{aligned}
$$

Namely,

$$
\begin{aligned}
\left|Y_{k}\right|^{2}+ & \lambda_{1} h\left|X_{k}\right|^{2}+\lambda_{2} h \sum_{i=k-m+1}^{k-1}\left|X_{i}\right|^{2}+\lambda_{4} h \sum_{i=k-m}^{k-1}\left|X_{i}\right|^{2} \\
\leq & \left|Y_{0}\right|^{2}+\lambda_{1} h\left|X_{0}\right|^{2}+\lambda_{2} h \sum_{i=-m+1}^{-1}\left|X_{i}\right|^{2}+\lambda_{4} h \sum_{i=-m}^{-1}\left|X_{i}\right|^{2} \\
& -\left(\lambda_{1}-\lambda_{3}-\lambda_{2}-\lambda_{4}\right) h \sum_{i=0}^{k-1}\left|X_{i}\right|^{2}+M_{k}
\end{aligned}
$$




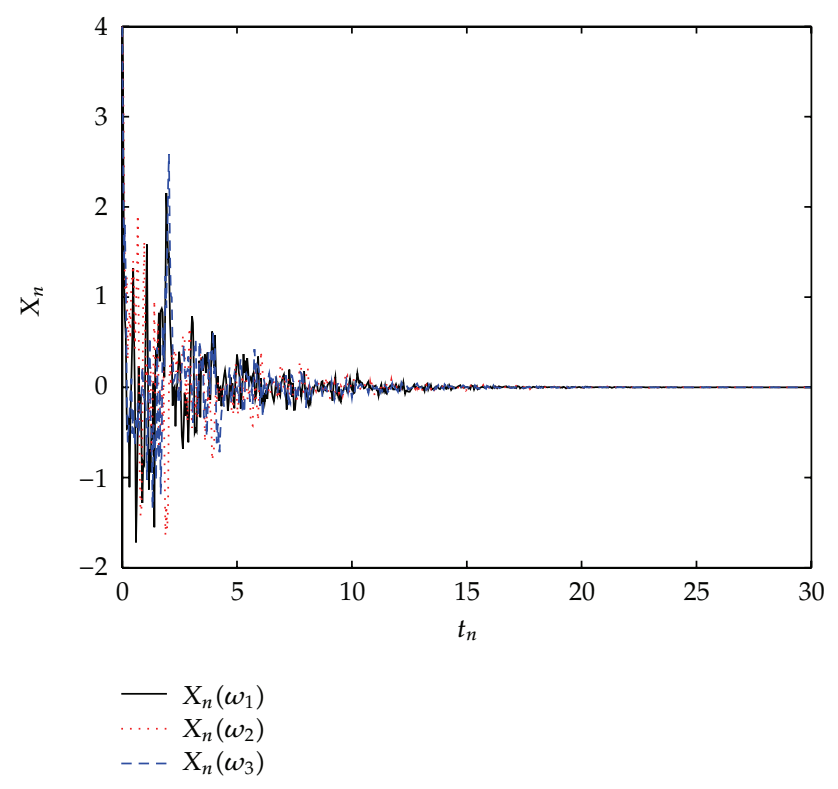

Figure 1: Almost surely asymptotic stability of the EM approximate solution $X_{n}$ with the stepsize $h=1 / 25$.

where $M_{k}=\sum_{i=0}^{k-1} m_{i}$. By [18], $M_{k}$ is a martingale with $M_{0}=0$. From (3.3), we obtain that

$$
\lambda_{1}-\lambda_{3}-\lambda_{2}-\lambda_{4}>0
$$

Using Lemma 2.2 yields

$$
\lim _{k \rightarrow \infty} \sum_{i=0}^{k-1}\left|X_{i}\right|^{2}<\infty \quad \text { a.s., } h>0 .
$$

Then, we conclude that

$$
\lim _{k \rightarrow \infty}\left|X_{k}\right|^{2}=0 \quad \text { a.s., } h>0 \text {. }
$$

The proof is completed.

\section{Numerical Experiments}

In this section, we present numerical experiments to illustrate the theoretical results presented in the previous sections. 


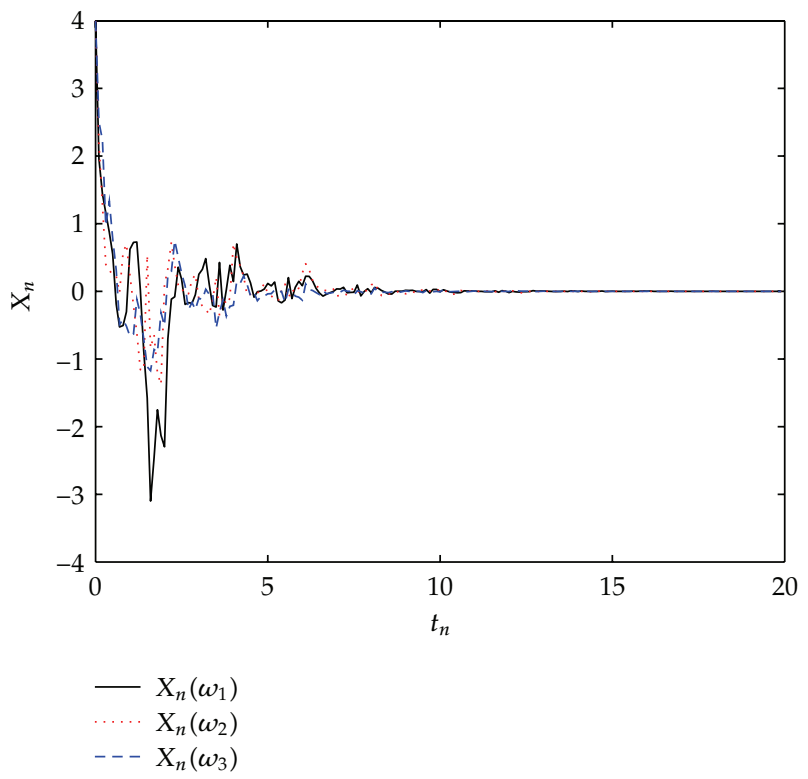

Figure 2: Almost surely asymptotic stability of the BEM approximate solution $X_{n}$ with the stepsize $h=0.1$.

Consider the following scalar linear problem

$$
\begin{gathered}
d\left[x(t)-\frac{1}{2} \sin (x(t-2))\right]=(-8 x(t)+\sin (x(t-2))) d t+x(t-2) d B(t), \quad t \geq 0, \\
x(t)=t+1, \quad-2 \leq t \leq 0 .
\end{gathered}
$$

For test (6.1), we have that $L=72, \lambda_{1}=11, \lambda_{2}=4, \lambda_{3}=0$, and $\lambda_{4}=1$. By Theorem 3.2, the exact solution to (6.1) is almost surely asymptotically stable.

Theorem 4.2 shows that the EM approximate solution to (6.1) can preserve the almost surely asymptotic stability of exact solutions for $h<1 / 24$. In Figure 1 , we compute three different paths $\left(X_{n}\left(\omega_{1}\right), X_{n}\left(\omega_{2}\right), X_{n}\left(\omega_{3}\right)\right)$ using EM (4.1) to approximate (6.1) with the stepsize $h=1 / 25$. Figure 1 shows that $X_{n}\left(\omega_{1}\right), X_{n}\left(\omega_{2}\right), X_{n}\left(\omega_{3}\right)$ are asymptotically stable. Theorem 5.1 shows that the BEM approximate solution to (6.1) reproduces the almost surely asymptotic stability of exact solutions for any $h>0$. In Figure 2, three different paths $\left(X_{n}\left(\omega_{1}\right), X_{n}\left(\omega_{2}\right), X_{n}\left(\omega_{3}\right)\right)$ are computed by using the BEM (5.1) to approximate (6.1) with the stepsize $h=0.1$. Figure 2 demonstrates that these paths are asymptotically stable.

\section{Conclusions}

This paper deals with the almost surely asymptotic stability of Euler-type methods for NSDDEs by using the discrete semimartingale convergence theorem. We show that the EM reproduces the almost surely asymptotic stability of exact solutions to NSDDEs under an additional linear growth condition. If we assume the BEM is well defined, the BEM can also preserve the almost surely asymptotic stability without the additional linear growth condition. 


\section{Acknowledgments}

The authors would like to thank the referees for their helpful comments and suggestions. This work is supported by the NSF of China (no. 11071050).

\section{References}

[1] V. B. Kolamnovskii and V. R. Nosov, Stability and Periodic Modes of Control Systems with Aftereffect, Nauka, Moscow, Russia, 1981.

[2] X. Mao, Stochastic Differential Equations and Their Applications, Horwood Publishing Series in Mathematics \& Applications, Horwood Publishing, 1997.

[3] D. Z. Liu, G. Y. Yang, and W. Zhang, "The stability of neutral stochastic delay differential equations with Possion jumps by fixed poins," Journal of Computational and Applied Mathematics, vol. 235, pp. 3115-3120, 2011.

[4] X. Mao, Y. Shen, and C. Yuan, "Almost surely asymptotic stability of neutral stochastic differential delay equations with Markovian switching," Stochastic Processes and Their Applications, vol. 118, no. 8, pp. 1385-1406, 2008.

[5] B. J. Yin and Z. H. Ma, "Convergence of the semi-implicit Euler method for neutral stochastic delay differential equations with phase semi-Markovian switching," Applied Mathematical Modelling, vol. 35, pp. 2094-2109, 2011.

[6] S. B. Zhou and F. K. Wu, "Convergence of numerical solutions to neutral stochastic delay differential equations with Markovian switching," Journal of Computational and Applied Mathematics, vol. 229, no. 1, pp. 85-96, 2009.

[7] S. Gan, H. Schurz, and H. Zhang, "Mean square convergence of stochastic $\theta$-methods for nonlinear neutral stochastic differential delay equations," International Journal of Numerical Analysis and Modeling, vol. 8, no. 2, pp. 201-213, 2011.

[8] C. T. H. Baker and E. Buckwar, "Exponential stability in $p$-th mean of solutions, and of convergent Euler-type solutions, of stochastic delay differential equations," Journal of Computational and Applied Mathematics, vol. 184, no. 2, pp. 404-427, 2005.

[9] M. Liu, W. Cao, and Z. Fan, "Convergence and stability of the semi-implicit Euler method for a linear stochastic differential delay equation," Journal of Computational and Applied Mathematics, vol. 170, no. 2, pp. 255-268, 2004.

[10] D. J. Higham, "Mean-square and asymptotic stability of the stochastic theta method," SIAM Journal on Numerical Analysis, vol. 38, no. 3, pp. 753-769, 2000.

[11] Y. Saito and T. Mitsui, "Stability analysis of numerical schemes for stochastic differential equations," SIAM Journal on Numerical Analysis, vol. 33, no. 6, pp. 2254-2267, 1996.

[12] S. Pang, F. Deng, and X. Mao, "Almost sure and moment exponential stability of Euler-Maruyama discretizations for hybrid stochastic differential equations," Journal of Computational and Applied Mathematics, vol. 213, no. 1, pp. 127-141, 2008.

[13] D. J. Higham, X. Mao, and C. Yuan, "Almost sure and moment exponential stability in the numerical simulation of stochastic differential equations," SIAM Journal on Numerical Analysis, vol. 45, no. 2, pp. 592-609, 2007.

[14] Y. Saito and T. Mitsui, "T-stability of numerical scheme for stochastic differential equations," in Contributions in Numerical Mathematics, vol. 2, pp. 333-344, World Scientific Series in Applicable Analysis, 1993.

[15] W. Q. Wang and Y. P. Chen, "Mean-square stability of semi-implicit Euler method for nonlinear neutral stochastic delay differential equations," Applied Numerical Mathematics, vol. 61, pp. 696-701, 2011.

[16] A. N. Shiryayev, Probablity, Springer, Berlin, Germany, 1996.

[17] A. Rodkina and H. Schurz, "Almost sure asymptotic stability of drift-implicit $\theta$-methods for bilinear ordinary stochastic differential equations in $\mathbb{R}^{1}$," Journal of Computational and Applied Mathematics, vol. 180, no. 1, pp. 13-31, 2005.

[18] F. Wu, X. Mao, and L. Szpruch, "Almost sure exponential stability of numerical solutions for stochastic delay differential equations," Numerische Mathematik, vol. 115, no. 4, pp. 681-697, 2010.

[19] Q. Li and S. Gan, "Almost sure exponential stability of numerical solutions for stochastic delay differential equations with jumps," Journal of Applied Mathematics and Computing. In press. 


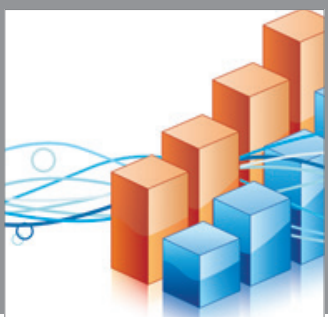

Advances in

Operations Research

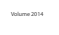

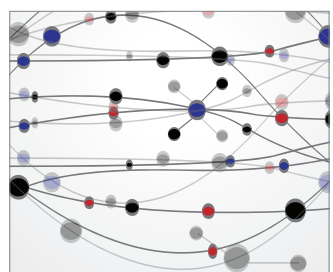

\section{The Scientific} World Journal
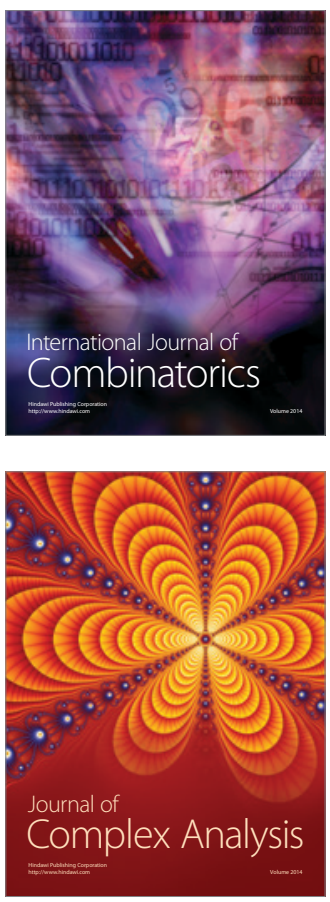

International Journal of

Mathematics and

Mathematical

Sciences
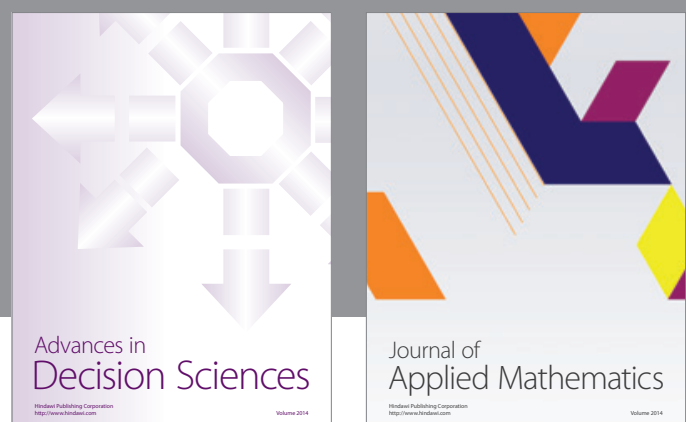

Journal of

Applied Mathematics
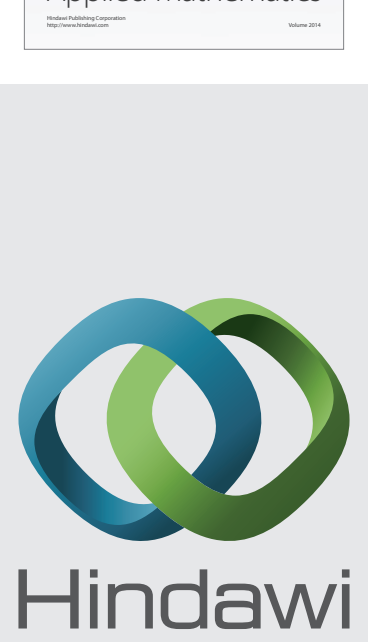

Submit your manuscripts at http://www.hindawi.com
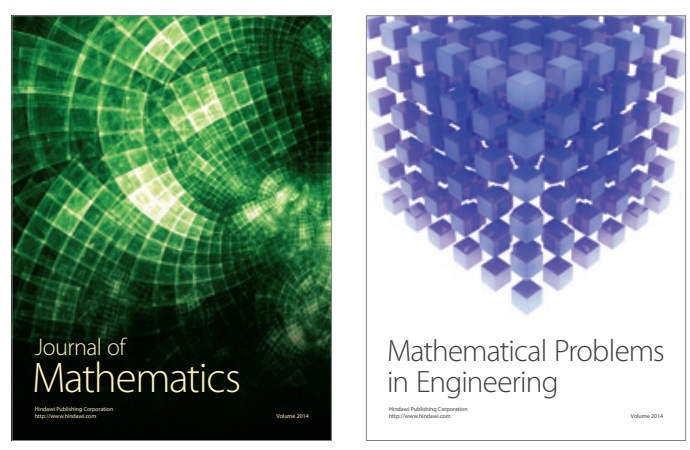

Mathematical Problems in Engineering
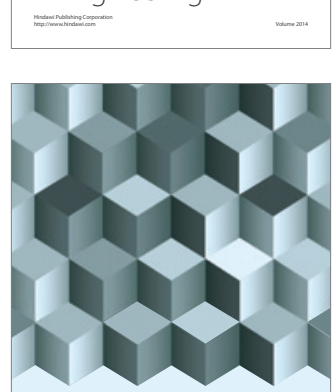

Journal of

Function Spaces
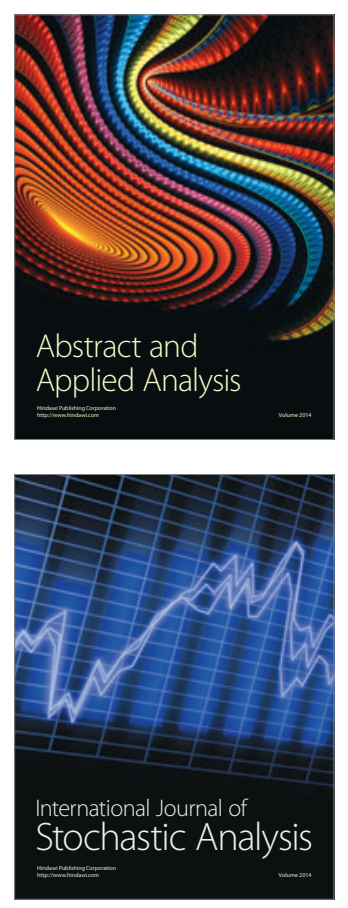

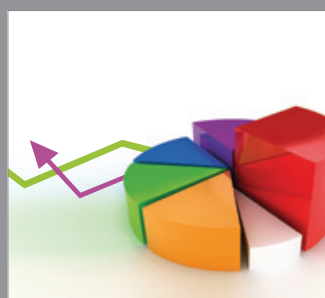

ournal of

Probability and Statistics

Promensencen
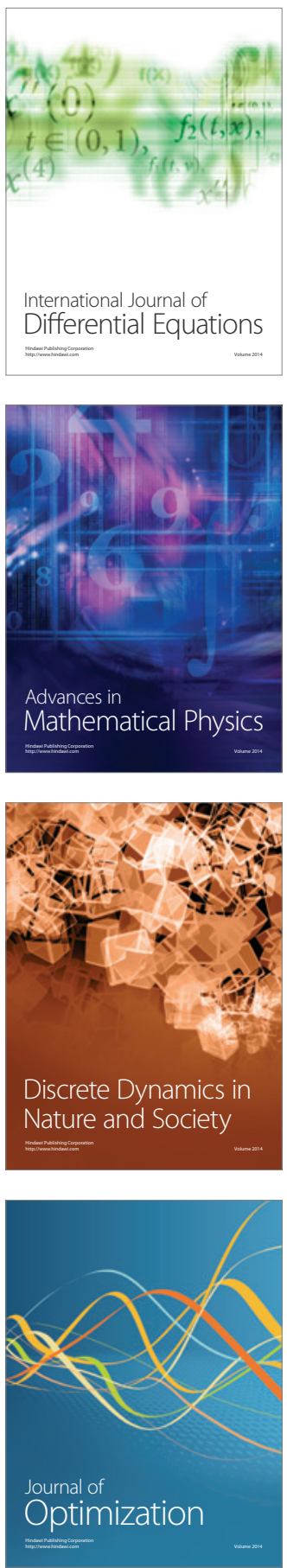